Revue musicale OICRM

revue musicale oicrm

\title{
Mes doigts sont-ils à moi ?
}

\section{Michel Chion}

Volume 5, numéro 1, 2018

Ce que le cinéma nous apprend de la musique

URI : https://id.erudit.org/iderudit/1044440ar

DOI : https://doi.org/10.7202/1044440ar

Aller au sommaire du numéro

\section{Éditeur(s)}

Observatoire interdisciplinaire de création et recherche en musique (OICRM)

\section{ISSN}

2368-7061 (numérique)

Découvrir la revue

\section{Citer cet article}

Chion, M. (2018). Mes doigts sont-ils à moi ? Revue musicale OICRM, 5(1), 1-6. https://doi.org/10.7202/1044440ar

\section{Résumé de l'article}

L'auteur s'attache à une série de films mettant en scène des musiciens classiques dont la carrière est traversée par leurs problèmes sentimentaux ou leurs névroses ; il s'intéresse particulièrement au mélodrame The Seventh Veil (Robert Compton-Bennett, 1945) dont l'action conjugue les motifs du pianiste ténébreux et de la pianiste-victime. Analysant le montage des corps des interprètes (celui de l'actrice, celui de sa doublure), il montre comment ce film dit le faux quand il fait croire par des inserts de mains d'une virtuose que c'est l'actrice qui joue du piano, et comment, en même temps, il dit le vrai par le faux quand il illustre métaphoriquement que les mains de quelqu'un ne lui appartiennent pas complètement : elles sont dressées, menées, mais aussi tentées, happées, entraînées par une autre force dont le reste du corps ne trahit rien. 


\title{
Mes doigts sont-ils à moi ?
}

\author{
Michel Chion
}

\begin{abstract}
Résumé
L'auteur s'attache à une série de films mettant en scène des musiciens classiques dont la carrière est traversée par leurs problèmes sentimentaux ou leurs névroses ; il s'intéresse particulièrement au mélodrame The Seventh Veil (Robert Compton-Bennett, 1945) dont l'action conjugue les motifs du pianiste ténébreux et de la pianiste-victime. Analysant le montage des corps des interprètes (celui de l'actrice, celui de sa doublure), il montre comment ce film dit le faux quand il fait croire par des inserts de mains d'une virtuose que c'est l'actrice qui joue du piano, et comment, en même temps, il dit le vrai par le faux quand il illustre métaphoriquement que les mains de quelqu'un ne lui appartiennent pas complètement : elles sont dressées, menées, mais aussi tentées, happées, entraînées par une autre force dont le reste du corps ne trahit rien.
\end{abstract}

Mots clés : pianiste ; main ; prise de vue ; montage ; pulsion.

\begin{abstract}
The author focuses on a series of films featuring classical musicians whose career is stressed by their emotional problems or neuroses; he is particularly interested in The Seventh Veil melodrama (Robert Compton-Bennett, 1945) whose action combines the types of the dark pianist and the pianist-victim. Analyzing the editing of the bodies of the performers (that of the actress, the one of her understudy), he shows how this film says the false when, using hand inserts of a virtuoso, it makes believe that the actress plays the piano, and how, at the same time, it tells the truth by the false when it illustrates metaphorically that the hands of someone do not entirely belong to him: they are erected, led, but also tempted, caught, driven by another force whose rest of the body betrays nothing.
\end{abstract}

Keywords: pianist; hand; camera angle; editing; urge.

Malgré son titre émoustillant, The Seventh Veil (1945), réalisé en Grande-Bretagne par Robert Compton-Bennett, n'a rien à voir avec la danse de Salomé et n'offre au spectateur aucune image de chair dénudée à découvrir, sinon le décolleté, les bras et les mains d'une belle actrice. Les voiles qu'il s'agit de lever sont mentaux. Il s'agit d'un de ces mélodrames mettant en scène des musiciens classiques dont la carrière est traversée par leurs problèmes sentimentaux ou leurs névroses, mélodrames dont la fréquence au cours des années 40 , notamment dans le cinéma américain, permet de parler sinon d'une vague, au moins d'une vaguelette. Vaguelette timide dans d'autres 
pays : comme exception en France, je citerais le film assez pâle d'Henri Decoin Les Amoureux sont seuls au monde (1948), avec Louis Jouvet jouant un compositeur tombé amoureux d'une jeune pianiste et dont l'épouse se suicide. Aux USA, les œuvres de ce genre furent bien plus nombreuses. Citons, entre autres : Intermezzo, a Love Story (Gregory Ratoff, 1940, avec Ingrid Bergman en pianiste et Leslie Howard en violoniste), Song of Russia (Gregory Ratoff à nouveau, 1944 ; ici une pianiste russe tombe amoureuse d'un chef d'orchestre américain - l'action se déroule avant la guerre froide !), Deception (Irving Rapper, 1945, dans lequel Bette Davis interprète une pianiste amoureux d'un violoncelliste), Humoresque (Jean Negulesco, 1947, où John Garfield, doublé par Isaac Stern, joue du violon), Rhapsody (Charles Vidor, 1954 ; ici Elisabeth Taylor violoniste est partagée entre un pianiste et un violoniste), et pour clore cette liste abrégée, I've always loved you (Frank Borzage, 1946) portant à nouveau sur une pianiste éprise d'un chef d'orchestre. Dans ces histoires, les rôles d'artistes du clavier reviennent souvent aux actrices. Lorsque c'est un homme, sa maîtrise de l'instrument est plutôt montrée sous un jour inquiétant, voire satanique. Ainsi, dans l'adaptation par Albert Lewin du Portrait de Dorian Gray, d'Oscar Wilde, toujours en 1945, le jeune séducteur ténébreux Dorian (Hurd Hatfield) joue, tel un thème personnifiant sa damnation, l'extraordinaire Prélude en ré mineur de Chopin, celui qui se termine - et achève tout le recueil des 24 Préludes - par un ré à l'extrême grave, au terme de vertigineuses plongées. Encore une fois, ici, derrière l'acteur, il y a une doublure pour l'interprétation, sinon pour les mains, dans ce cas la pianiste Lela Simone, qui travaillait à l'époque pour la MGM.

Le pianiste fou que ses mains entraînent au pire est un thème familier du roman d'horreur : une histoire écrite par le français Maurice Renard, Les Mains d'Orlac, a même connu plusieurs adaptations à l'écran, et inspiré indirectement le mélodrame de Robert Florey bien nommé The Beast with Five Fingers (1946), qui tourne autour de l'assimilation main de pianiste (un des personnages est un virtuose paralysé de la main droite) et main d'étrangleur. Dans d'autres cas, il s'agit d'un compositeur fou commettant des crimes lors de ses crises d'identité, comme avec Hangover Square (1945), réalisé par John Brahm. Pour terminer, sans épuiser le lecteur, cette liste destinée à montrer que la vaguelette des années 40 ressemblait presque à une vague, n'oublions pas que le film d'Alfred Hitchcock, The Rope (1948), fait d'un des deux jeunes dandys étrangleurs (celui joué par Farley Granger) un pianiste, dont le plaisir est de jouer le Mouvement Perpétuel de Poulenc, caractérisé par sa fixité de tempo inquiétante.

Précisément, l'action de The Seventh Veil, sur lequel nous voudrions nous attarder, conjugue les motifs du pianiste ténébreux (ici, un infirme jaloux et tyrannique, joué par James Mason) et de la pianiste-victime, qui est ici sa créature docile. Cette jeune femme, nommée Francesca Cunningham (Ann Todd), subit donc son emprise, et par amour elle se laisse former, dresser, dresser impitoyablement pour essayer de devenir la grande soliste qu'il voit en elle. Par ailleurs, il ne cesse de lui recommander de faire attention à ses mains, et le film constitue un festival de gros plans de mains, nues ou gantées, au repos ou en action. Ce sont justement ces précieuses mains que dans un moment de colère James Mason frappe avec sa canne, plongeant dans le désespoir la jeune femme, persuadée dès lors qu'elle ne pourra plus jouer de sa vie. Elle tente de mettre fin à ses jours et se jette dans les flots. Sauvée de la noyade, elle est confiée aux soins d'un curieux thérapeute, le docteur Larsen (Herbert Lom), qui lui fait revivre 
ses traumatismes dans des séances d'hypnose, et lui redonne, sinon l'usage de ses mains de virtuose, du moins confiance en la vie.

Différents thèmes se croisent ici : notamment une variante du motif de Pygmalion, traité dans la pièce homonyme de George Bernard Shaw, celle-là même qui a inspiré la comédie musicale My Fair Lady et le film qui en a été tiré, et le thème psychanalytique fréquent à l'époque (on le trouve aussi dans Spellbound avec Ingrid Bergman et Gregory Peck, tourné la même année par Hitchcock).

Le film de Compton Bennett comporte plusieurs scènes de concert symphonique : dans l'une d'elles, et devant un public très chic, la pauvre Francesca, qui ne cesse de se tordre les mains quand elle ne les a pas sur le clavier, vient à bout du Concerto pour piano et orchestre en la mineur de Grieg, sous l'œil froid du chef d'orchestre (joué par Muir Mathieson lui-même, qui a dirigé beaucoup de musiques de film anglaises, et ici se montre devant la caméra). Le spectateur assiste à l'habituelle succession de plans où l'artiste est filmé sous différents axes avec différentes grosseurs. Tantôt Ann Todd filmée de face remue les bras tandis que ses mains sont cachées par le clavier, et l'on se doute bien qu'elle se contente de mimer le jeu. Tantôt nous la voyons de trois quarts dos parcourir le clavier, et l'on admire la synchronisation globale de ses bras sinon de ses doigts, avec ce que nous entendons ; tantôt les inserts de deux mains agiles filmées de près confirment la synchronisation - et le spectateur le plus innocent se doute bien que dans ce cas, les doigts ne sont plus les siens ; d'ailleurs, c'est Eileen Joyce qui a joué la partie de piano, appris à Ann Todd ses mouvements de bras, et prêté ses mains, fort belles d'ailleurs et fines, aux gros plans (Davis 2001, p. 120).

L'insert de ce type pourrait être considéré comme un procédé de tricherie - créant automatiquement ce que j'appellerai (l'adjectif sera expliqué plus loin) le soupçon horizontal du spectateur de film : cette partie du corps que je vois, isolée par le montage sur l'axe du temps, peut ne pas appartenir au personnage dont je suis l'histoire et à son interprète.

On sait d'ailleurs que l'insert a été abondamment utilisé dans le cinéma populaire, au cours des années 60-70, pour transformer en œuvres érotiques d'innocents films d'aventures. Dans les années 70, poussé par l'appréciation élogieuse qu'en faisait Jean-Pierre Bouyxou dans son livre sur le cinéma de science-fiction, j'avais vu le film d'aventures d'Antonio Margheriti La Terreur des Kirghizes, sans en retirer une impression précise, ni comprendre son rapport avec la SF. Deux ans plus tard, un ami de passage à Paris m'entraîne, sans que je me fasse prier, à entrer dans une salle vouée à des films qu'on n'appelait pas encore des films X, pour voir La Vie érotique d'Ursus. Dès le début, j'ai un étrange sentiment de " déjà vu », mais il me faut plusieurs minutes pour comprendre que je suis en train de regarder pour la deuxième fois le film de Margheriti, avec d'étranges plans rajoutés dans lesquels les personnages, ou plutôt leurs doublures-corps que le cadrage prive de tête, s'ébattent nus sous les tentes kirghizes. Dans ces scènes, l'image change de couleur, les raccords avec l'action principale sont grossiers, et personne ne peut, même à l'époque, être dupe des inserts.

C'est la même chose avec les films musicaux de la vague dont je parle : même si le raccord est beaucoup plus soigneux, le soupçon d'une doublure-main arrive immédiatement. Néanmoins, avant que n'arrivent la cassette, le DVD, les fichiers numériques, etc., où l'on peut stopper le film, le spectateur en salle accepte, dans le feu de l'action 
et le défilé continu de l'œuvre, de « jouer le jeu » et d'imputer aux personnages et à son interprète cette merveilleuse virtuosité.

Ce ne fut pas toujours le cas, au cours des années 70-80, les acteurs se firent au contraire un point d'honneur de refuser les « inserts de mains ", ce que l'on peut voir dans un film comme The Competition (1980) de Joel Oliansky, dans lequel Richard Dreyfuss et Amy Irving, concurrents dans un grand concours international, nous montrent qu'ils peuvent laisser voir leurs visages, leurs corps, leurs bras, leurs mains, dans les passages les plus difficiles, et que la synchronisation semble sans défaut. Ce n'est pas un hasard non plus si l'opposition entre la femme victime et créature, et l'homme manipulateur, a disparu : les deux concurrents sont de taille égale, et ils ont le même aplomb et la même agressivité pour gagner.

Aujourd'hui, les prouesses de la synthèse numérique créent un nouveau soupçon : on peut très bien " greffer » un bras et des mains virtuels sur un acteur filmé.

Revenons alors à ces poétiques et apparemment naïfs " inserts » de mains de virtuoses entre les plans d'un acteur. Ils disent une certaine vérité. Laquelle ? Que les mains de quelqu'un ne lui appartiennent pas complètement : elles sont dressées, menées, mais aussi tentées, happées, entraînées par une autre force dont le reste du corps ne trahit rien. Elles incarnent notamment ce que la psychanalyse appelle les pulsions partielles - ces envies d'agression, de prédation, de rapt, celles-là mêmes que le matraquage publicitaire cherche à éveiller chez nous lorsque, dans un magasin, nous nous emparons d'un produit de vaisselle ou d'un yaourt plutôt que d'un autre... Les mains sont à la fois la partie de notre corps qui est le plus visiblement douée d'habileté, mais aussi la plus sujette à nous " échapper ». Ajoutons à cela le fait que l'animal humain est latéralisé, c'est-à-dire avec une asymétrie de l'aisance manuelle, et une individualisation de chaque main qui est d'ailleurs un mystère du point de vue de l'évolution.

À ce propos, celui qui écrit ces lignes est ce qu'on appelle un "gaucher contrarié ». J'ai en effet été - c'était banal en France dans ma génération - un de ces gauchers de naissance qu'on dressait à écrire de la main droite, leur laissant l'usage de la main gauche pour toutes les autres actions quotidiennes réservées à une seule main.

Le thème du dressage digital fait inévitablement penser à une charmante comédie musicale de 1953, devenue " culte », The 5000 Fingers of Dr T., qui est comme la parodie de ces mélodrames pianistiques évoqués plus haut. Réalisée par Roy Rowland, cette œuvre poétique et plaisante raconte la plongée d'un petit garçon, sous l'emprise de sa mère et d'un professeur de piano tyrannique, dans un monde de rêves où le Dr T. dirige une sorte de bagne à petits virtuoses.

Au début du film, la mère du garçonnet lui fait jouer et chanter cette chanson volontairement niaise, qui prend pour sujet les dix doigts que le pianiste met en action :

Ten little dancing maidens, dancing all so fine!

Ten happy little fingers and they're mine all mine

They're mine, they're mine

Now isn't that just fine?

No three, not five, not seven and not nine,

But ten all dancing straight in line!

And all of them are mine, all mine.

Oh yes, they're mine, all mine! 
J'ai toujours ressenti ce que ce "they're mine ", "ils sont à moi, ils m'obéissent », est, plutôt qu'une constatation neutre, l'expression forcenée d'un déni. En réalité, nous le savons bien, ces dix doigts ne nous appartiennent pas, soit qu'ils se mettent à obéir à une partition ou à des mécanismes automatisés (fort utiles d'ailleurs dans l'usage quotidien... et dans l'écriture), soit qu'ils expriment la révolte des pulsions partielles.

À ce stade, nous ne sommes plus dans la musique « pure ». Mais c'est précisément le charme du cinéma que tout sujet qu'il traite se mette à devenir emblématique ou métaphorique. De même que le thème de l'orchestre symphonique devient presque automatiquement une parabole de la démocratie en crise et en risque de délitement (dans le Prova d'orchestra, 1978, de Fellini), ou bien des mérites comparés de la tyrannie et de l'autorité habile (dans Le chef d'orchestre, 1979, de Wajda) - de la même façon les mélodrames avec pianiste parlent toujours d'autre chose. D'ailleurs, la musique elle-même s'ouvre toujours sur autre chose, et par ailleurs cet article traite de cinéma : or un film est un cadre dont les propriétés sont de morceler dans le temps et dans l'espace et de recombiner (même les films " en un seul plan ", comme The Rope ou plus récemment Victoria, morcellent l'espace par le cadrage rectangulaire).

Le cinéma dit le faux quand il fait croire par des inserts de mains que c'est Ann Todd qui joue du piano, et en même temps, il dit le vrai par le faux quand il illustre métaphoriquement par le montage et le contraste entre le visage tendu de l'héroïne et ses mains agiles, sa division fondamentale, par son déchirement.

Une des scènes les plus troublantes de The Seventh Veil est celle où, pour rééduquer sa patiente, Larsen lui fait écouter en état d'hypnose un disque de la Sonate pathétique qu'elle a gravé jadis - sur le label His Master's Voice, tout un programme - et essaie de lui faire bouger ses doigts sur le clavier en se synchronisant avec son double enregistré. Or, Francesca n'arrive que maladroitement à se superposer à elle-même ; elle échappe alors à ce que j'appelle le soupçon vertical, celui d'une post-synchronisation (ou d'un playback) toujours pressentie par le spectateur, même quand il a affaire à ce qu'on appelle le « son direct».

Tout comme les spectateurs des premiers films muets utilisant le montage en étaient très conscients, et avaient le soupçon horizontal en alerte - quelques années plus tard, ceux des films parlants du début étaient, à l'inverse, méfiants sur l'appartenance des voix au corps, et des corps aux instruments. Loin d'être naïfs, ils éprouvaient à nu le "soupçon vertical ". Soit parce que ces films racontaient souvent des histoires de ventriloquie, de voix usurpée, de sifflotement échappant au siffleur, d'homme invisible - soit parce qu'il s'agissait de dessins animés musicaux dans lesquels un Mickey à huit doigts et sa compagne Minnie jouent du piano avec un synchronisme parfait, mais rendu suspect par le principe même du dessin (The Birthday Party, 1930).

En 1946, peu de temps après la sortie et le succès de The Seventh Veil, un film américain de William Wyler, très aimé aux USA, mais peu connu des cinéphiles européens The Best Years of Our Lives, donne un sens poignant à la question du synchronisme pianistique. Cette œuvre de près de trois heures raconte le retour au pays de plusieurs soldats américains après la Deuxième Guerre mondiale, de ceux qui ont survécu, en tout cas : certains sont intacts, d'autres mutilés, notamment un certain Homer, joué par un amputé authentique, Harold Russell, dont les mains sont remplacées par des crochets habilement conçus pour lui permettre de se saisir d'objets 
(pour s'habiller et se déshabiller, il lui faut toutefois l'aide de sa femme). Le film a l'audace de le confronter à un personnage de pianiste de bar, interprété par Hoagy Carmichael.

Ce pianiste-compositeur, auteur de chansons aussi mémorables que Stardust, est apparu sur les écrans dans plusieurs films, comme To Have and Have not, de Howard Hawks d'après Hemingway, où son personnage s'appelle Cricket et où il accompagne une Laurent Bacall de vingt ans, ou bien The Young Man with a Horn (1950), réalisé par Michael Curtiz, dans lequel il incarne l'ami d'un trompettiste génial joué par Kirk Douglas, et dont la vie s'inspire de celle de Bix Beiderbecke. Dans une scène marquante du film de Wyler, Hoagy propose à Homer de jouer avec ses crochets quelques notes sur le piano à ses côtés, formant avec lui un duo insolite, et qu'on pourrait juger choquant : du quatre mains, mais pour dix doigts et deux crochets. Pourquoi en effet quelqu'un qui n'a plus de mains ne pourrait-il pas s'amuser à taper sur des touches de piano?

Là encore cependant, se glisse le soupçon vertical (simultané) : Carmichael et Russell peuvent très bien, au tournage, avoir joué en playback sur leur propre son.

Sans jeu de mots, en effet, son au cinéma = soupçon. Aujourd'hui, nous l'avons dit plus haut, avec le numérique, le soupçon change de nature et se diffuse partout. On remarque d'ailleurs que le montage, la succession rapide de plans distincts, règne de plus belle ; le soupçon horizontal continuer de nous parler toujours de notre morcellement : ce que faisaient, à leur façon, les films des années 40 que nous avons évoqués.

BiBLIOGRAPHIE

Davis, Richard, Eileen Joyce: A Portrait, Fremantle, Fremantle Arts Centre Press, 2001. 